Report

\title{
RhoC induces differential expression of genes involved in invasion and metastasis in MCF10A breast cells
}

\author{
Mei $\mathrm{Wu}^{1,3}$, Zhi-Fen $\mathrm{Wu}^{1,3}$, Chandan Kumar-Sinha ${ }^{2,4}$, Arul Chinnaiyan ${ }^{2}$, and Sofia D. \\ Merajver $^{1,3}$ \\ ${ }^{1}$ Department of Internal Medicine, Division of Hematology and Oncology, ${ }^{2}$ Department of Pathology, \\ ${ }^{3}$ Comprehensive Cancer Center, University of Michigan, Ann Arbor, MI, USA; ${ }^{4}$ Present Address: Institute of \\ Bioinformatics, Unit1, Bangalore, India
}

Key words: breast cancer, invasion, MCF10A, microarray, motility, RhoC

\begin{abstract}
Summary
Inflammatory breast cancer (IBC) is the most deadly form of breast cancer in humans presumably due to its ability to metastasize from its inception. In our laboratory, overexpression of RhoC GTPase was observed to be specific for IBC tumors, but not for stage-matched, non-IBC tumors. RhoC is known to contribute to an IBC-like phenotype in HPV-E6E7 immortalized breast cells. To further study the effect of RhoC overexpression on IBC metastasis, we generated stable transfectants of spontaneous immortalized mammary epithelial cells (MCF10A) overexpressing wild-type RhoC or a constitutively active RhoC mutant (G14V). Both the RhoC wild type and the G14V transfectants were highly invasive and proliferated more rapidly compared to vector-only control clones. Overexpression of RhoC led to an increase in actin stress fiber and focal adhesion contact formation. Comparative microarray analysis of these clones further revealed that RhoC overexpression upregulated 108 genes whereas seven genes were down-regulated. We have further verified by quantitative RT-PCR that genes involved in cell proliferation, invasion/adhesion, and angiogenesis were modulated by RhoC. This work suggests strong candidates for the downstream oncogenic functions of RhoC.
\end{abstract}

Abbreviations: CXCL1: CXC chemokine ligand 1; EGF: epidermal growth factor; GAPDH: glyceraldehyde-3phosphate dehydrogenase; GEF: guanine nucleotide exchange factor; HA: influenza virus hemagglutinin epitope; HPV: human papilloma virus; IBC: inflammatory breast cancer; IGF: insulin-like growth factor; IGF-BP: IGFbinding protein; RT-PCR: reverse transcriptase-PCR; TGF- $\beta$ : transforming growth factor- $\beta$; VEGF: vascular endothelial growth factor; WT: wild-type

\section{Introduction}

Primary IBC is the most lethal form of breast cancer and accounts for approximately $6 \%$ of new breast cancer cases annually in the United States $[1,2]$. IBC is clinically well characterized by a very rapid onset of skin changes such as erythema, skin nodules, dimpling of the skin (termed 'peau d'aurange'), and nipple retraction, typically within six months [1-4]. IBC cells acquire metastatic capabilities early in tumor formation; nearly all women with IBC have tumor emboli that are present in the dermal lymphatics at the time of diagnosis, and over one-third have distant metastases [3, 4]. In spite of modern multimodality treatments, the 5-year disease-free survival is less than $45 \%$, thus making IBC the most deadly form of locally advanced breast cancer $[3,4]$.

In our laboratory, we sought to identify the genetic basis of IBC development and progression [5]. Overexpression of RhoC GTPase [6] and down-regulation of WISP3 [7] are characteristic and specific for IBC, in work performed on SUM149, an IBC cell line [8]. Moreover, overexpression of RhoC GTPase was identified in $90 \%$ of IBC archival tumor samples, but not in stage III non-IBC tumors [5]. 
RhoC belongs to the Ras superfamily of small GTPase, which includes RhoA and RhoB (homologous to RhoC) as well as many others, including Rac and $\mathrm{Cdc} 42$ [9]. Upregulation of RhoC plays important roles in metastasis not only in IBC, but also in a plethora of malignant neoplasms including bladder [10], ovary [11], pancreas [12], and skin (melanoma) [13]. However, RhoC signaling and its downstream molecular targets involved in IBC progression are still poorly understood.

In order to gain further insight into RhoC downstream signaling, we have developed breast-specific cellular reagents that would allow us to study the gene expression changes caused by RhoC upregulation. Stable transfectants of wild-type RhoC (RhoC-WT) and a constitutively active RhoC mutant (G14V) were generated in human mammary epithelial cells. We performed oligonucleotide/cDNA microarray comparative analysis of these clones and vector-only transfectants. Our laboratory had previously established stable wild-type RhoC transfectants in HPV-E6/E7 immortalized human mammary epithelial cells (termed HME-E6/E7), which acquired an aggressive IBC-like phenotype, including high motility and invasiveness, ability to grow on soft agar, and ability to form tumors in nude mice [6].

For studies of gene expression changes, we aimed to develop cellular reagents that isolated RhoC overexpression as a genetic event, independent of viral immortalization of the parental cell line. In this study, we employed RhoC constructs (Guthrie cDNA Resource Center) which comprise only the coding region of RhoC with three HA-tags at the N-terminus in pcDNA3.1 (Invitrogen, Carlsbad, CA). To avoid potential phenotype changes associated with immortalization, we used MCF10A cells as the parental substrate. MCF10A was derived from the proliferative epithelium of a fibrocystic lesion [14]. Importantly, biological assays showed that the MCF10A-RhoC WT and the G14V transfectants exhibited enhanced invasion and cell proliferation quantitatively comparable to the original HME E6/E7 clones. The formation of stress fibers and focal adhesion in these cells was significantly increased, which is also characteristic in cells overexpressing Rho family proteins [9], further supporting that over-expression of RhoC morphologically transformed MCF10A cells.

Overall, the array analysis of our wild-type/G14V RhoC clones lends strong support for a role of RhoC in invasion and metastasis. The data showed increased expression in 105 genes, and decreased expression in seven genes. We confirmed RhoC-induced differential expression in several genes using RT-PCR. Here we present the first summary data of genome-wide expression changes induced by RhoC overexpressionmediated transformation of breast cells.

\section{Materials and methods}

\section{Cell culture}

MCF10A cells [14] were cultured in 1:1 mixture of Ham's F12 (Mediatech, Herndon, VA) and DMEM containing L-glutamine (Invitrogen, Carlsbad, CA), supplemented with 5\% horse serum (Invitrogen, Carlsbad, CA), EGF, cholera toxin, insulin, hydrocortisone (Sigma, St. Louis, MO) at $37^{\circ} \mathrm{C}$ under $10 \%$ $\mathrm{CO}_{2}$. MCF10A cells are cytokeratin positive luminal cells, which correlates with the histologic type of the IBC SUM149 [15]. MCF10A cells were transfected with either pcDNA3.1 (Invitrogen, Carlsbad, CA), pcDNA3.1-RhoC wildtype or with pcDNA3.1RhoC constitutively active G14V mutant (Guthrie cDNA Resource Center, http://www.cdna.org), using FuGene 6 (Roche Applied Science, Indianapolis, IN). Stable transfectants were developed as previously described [6] and maintained in the medium supplemented with $600 \mu \mathrm{g} / \mathrm{ml} \mathrm{G} 418$ (Invitrogen, Carlsbad, CA).

\section{Western analysis}

Cells were washed in ice-cold phosphate-buffered saline, lysed in lysis buffer $(10 \%$ glycerol, $50 \mathrm{mM}$ Tris $\mathrm{pH} 7.4,100 \mathrm{mM} \mathrm{NaCl}, 1 \% \mathrm{NP}-40,2 \mathrm{mM} \mathrm{MgCl} 2$, $1 \mu \mathrm{g} / \mathrm{ml}$ leupeptin, $1 \mu \mathrm{g} / \mathrm{ml}$ aprotinin, $1 \mathrm{mM}$ PMSF) on ice for $5 \mathrm{~min}$, and then centrifuged for $5 \mathrm{~min}$ at $4^{\circ} \mathrm{C}$. Cleared lysates $(50 \mu \mathrm{g}$ of protein each) were subjected to SDS-PAGE and transferred to PVDF membrane. Western blots were performed as described [16] using the anti-RhoC rabbit polyclonal antibody [16], the anti-HA monoclonal antibody (Covance, Princeton, NJ) and anti- $\beta$-actin goat antibody (Sigma, St. Louis, MO) at dilutions of 1:1500, 1:1200 and 1:2000, respectively.

\section{Quantitative RT-PCR}

Total messenger RNA was isolated using Trizol reagent (Invitrogen, Carlsbad, CA) per the manufacturer's instruction. One microgram of total RNA was converted to cDNA using an avian myeloblastosis 
virus reverse transcription system (Promega, Madison, WI). A 100-mg aliquot of the resulting cDNA was amplified in a double PCR with $25 \mathrm{ng}$ each of HA/GAPDH-specific primers or with the sequencespecific primers for the genes of interest. PCR products were separated on a $1.2 \%$ agarose gel and imaged on an Alpha Image 950 documentation system (Alpha Innotech, San Leandro, CA). Densitometry of the images was performed using AlphaEaseFC (Alpha Innotech, San Leandro, CA).

\section{Cell staining of HA-tagged RhoC and rhodamine-phalloidin}

Cells were grown on chamber slides for $24 \mathrm{~h}$ and washed with PBS followed by fixation with $3 \%$ paraformaldehyde in PBS for $10 \mathrm{~min}$. After incubating in $0.1 \%$ Triton and blocking in $1 \%$ BSA, 2 Units of methanolic rhodamine-phalloidin stock (Molecular Probes, Eugene, OR) or 1:1000 dilution of the anti-HA monoclonal antibody (Covance, Princeton, NJ) in $200 \mu 1$ PBS were added to each coverslip and allowed to stain for $1 \mathrm{~h}$ at room temperature. The samples containing anti-HA were further stained with 1:400 dilution of Alexa Fluor 488-conjugated anti-mouse secondary antibody (Molecular Probes, Eugene, OR). All the slides were mounted by Gel/Mount (Biomedia Co., Foster City, CA). Cells were visualized under an Olympus fluorescence microscope.

\section{Monolayer growth rate}

Monolayer culture growth rate was determined by conversion of MTT (3-(4,5-dimethylthiazol-2-yl)-2,5diphenyltetrazolium bromide) (Sigma, St. Louis, MO) to a water-insoluble formazan by viable cells. Fivehundred cells in $200 \mathrm{ml}$ of medium were plated in 96-well plates and grown under normal conditions. Cultures were assayed at 1, 3, 5, 7, 9, and 11 days by adding $40 \mathrm{ml}$ of $5 \mathrm{mg} / \mathrm{ml}$ MTT and incubating for $1 \mathrm{~h}$ at $37^{\circ} \mathrm{C}$. The medium was aspirated and $100 \mathrm{ml}$ of DMSO (Sigma, St. Louis, MO) were added to lyse the cells and solubilized the formazan. Absorbance values of the lysates were determined on a Dynatech MR 5000 microplate reader at $595 \mathrm{~nm}$.

Quantitative invasion assay

of the stable transfectants

Invasion assays were performed on 24-well culture dishes per manufacturer's instructions (Chemicon International, Temecula, CA). The inserts within each dish are equipped with a polycarbonate membrane on the bottom coated with a thin layer of extracellular matrix (ECM) proteins. In brief, the inserts were rehydrated in serum-free media. Cells were washed in PBS and resuspended in serum-free medium at a concentration of $5 \times 10^{5}$ cells $/ \mathrm{ml}$ and $0.5 \mathrm{ml}$ of cell suspension was added to the insert. Wells in the culture dish were filled with serum-containing medium. The dish was incubated for $24 \mathrm{~h}$ at $37^{\circ} \mathrm{C}$ in a $10 \% \mathrm{CO}_{2}$ incubator. The cell suspension was aspirated and noninvasive cells were removed from the insert using a cotton swab. The inserts were stained with the staining solution for $20 \mathrm{~min}$, then rinsed in water. Stained cells were dissolved in $10 \%$ acetic acid, and measured OD at $595 \mathrm{~nm}$.

\section{Microarray procedures}

DNA microarray analysis of gene expression was carried out as previously described [17]. Briefly, purified PCR products, generated using clone inserts (sequence verified Human cDNA clones from ResGen, Carlsbad, CA) as template, were spotted onto polyL-lysine coated microscope slides using an Omnigrid robotic arrayer (GeneMachines, San Carlos, CA) equipped with quill-type pins (Majer Precision Engineering, Tempe, AZ) to generate 20,000 element human cDNA microarrays. Protocols for printing and post-processing of arrays are available in the public domain [18].

\section{Isolation of RNA, labeling of cDNA probes and hybridization to $c D N A$ arrays}

Approximately 4-6 million cells per $100-\mathrm{mm}$ tissue culture plate were harvested in Trizol (Invitrogen, Carlsbad, CA) and total RNA was isolated according to the manufacturer's protocol. An extra phenol-chloroform extraction was performed to improve the quality of RNA for microarray analyses. Total RNA was quantified, RNA integrity adjusted by denaturing-formaldehyde agarose gel electrophoresis, and stored in aliquots at $-80^{\circ} \mathrm{C}$ until ready to use. Forty micrograms of total RNA was routinely used as template for cDNA generation using reverse transcriptase (RT) (Invitrogen, Carlsbad, CA). Inclusion of amino allyl-dUTP (Sigma, St. Louis, MO) in the RT reaction allowed for subsequent fluorescent labeling of cDNA using mono-functional NHS ester dyes (Amersham Biosciences, Piscataway, NJ) as described [18]. For every hybridization, fluorescent 
cDNA probes were prepared from RNA from RhoCtransfected cells (coupled to Cy5 NHS-ester) and matched vector transfected reference cells (coupled to Cy3 NHS-ester). The labeled probes were then hybridized to the $20 \mathrm{~K}$ human cDNA microarrays at $65^{\circ} \mathrm{C}$ overnight as described [18]. Fluorescent images of hybridized microarrays were obtained using a GenePix 4000A microarray scanner (Axon Instruments, Union City, CA).

\section{Results}

Identification and confirmation of MCF10A-RhoC/G14V overexpressing clones

In order to isolate the MCF10A-RhoC-WT/G14V stable transfectants, quantitative RT-PCR and western analysis were conducted. Using primers specifically designed to amplify HA tags and their junction with RhoC, RT-PCR revealed a 5-fold increase in HA-RhoC mRNA in the MCF10A-RhoC transfectants (Figure 1(A)). The levels of RhoC expression correlated well with those of the primary SUM149 cell line and the previously described HME-RhoC transfectants $[5,6]$. Western analysis further confirmed that the RhoC protein levels in the MCF10A-RhoC cells are approximately two-fold higher than in the vectoronly controls (Figure 1(B)). For further verification, we also tested HA-RhoC protein levels and protein localization in the transfectants using immunofluorescence staining (Figure 2, HA MoAb). As expected, the MCF10A-RhoC clones stained very strongly with a monoclonal antibody against HA compared to the vector only control. Based on the RT-PCR and western analysis, we selected two clones each for both MCF10A-RhoC WT and MCF10A-G14V for our subsequent studies.

\section{Overexpression of RhoC in MCF10A-RhoC increased stress fiber formation, monolayer growth rates, and invasion}

The most prominent function of the Rho family proteins is to induce the assembly of actin and myosin filaments (stress fibers), to enhance focal adhesions, and to act collaboratively with Rac and Cdc42 in cell movement [9]. RhoC overexpression in HME E6/E7 is known to enhance formation of stress fibers [6]. In our new MCF10A-RhoC WT transfectants, we have likewise observed significant induction of the assembly
(A)

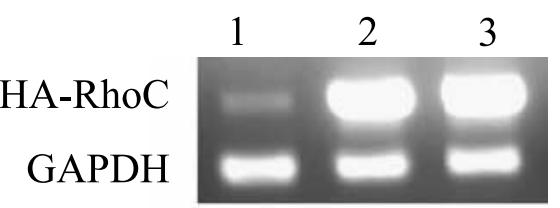

(B)

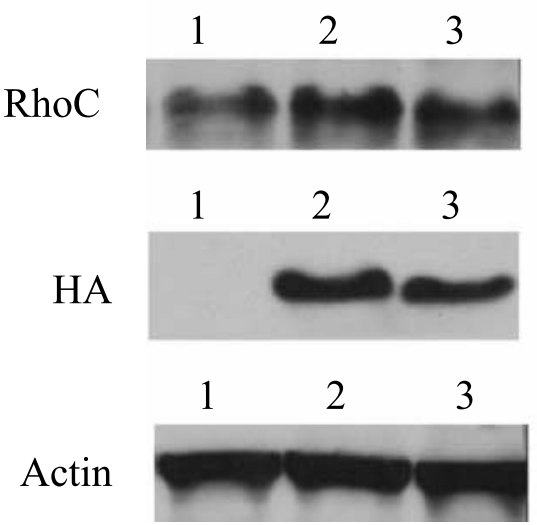

Figure 1. Expression of HA-RhoC in MCF10A-RhoC clones. (A) Quantitative RT-PCR was performed on mRNA extracts using primers specific for the HA-RhoC fusion gene, and GAPDH (control). MCF10A breast cells stably transfected with pcDNA3.1 (vector-only), pcDNA3.1-HA-RhoC WT (RhoC-WT) or pcDNA3.1-HA-RhoC G14V (RhoC-G14V). (B) RhoC protein expression was confirmed by western analysis using the anti-HA monoclonal antibody and the anti-RhoC polyclonal antibody. Cleared lysates $(50 \mu \mathrm{g}$ of protein each) were subjected to SDS-PAGE and transferred to PVDF membrane for western analysis. The protein levels of $\beta$-actin were used as loading controls.

of actin filaments, using rhodamine-phalloidin to specifically stain F-actin (Figure 2). The control cells were round and small; on the other hand, the RhoC WT cells stretched in polygonal shapes and formed multiple focal adhesion points (Figure 2).

The HME-E6/E7 RhoC clones had not shown significant increase of monolayer growth rate from the control cells [6]. Interestingly, the monolayer growth rate assay of the MCF10A-RhoC WT/G14V cells showed a two-fold increase in proliferating rates compared to the vector-only controls, in three independent experiments (Figure 3(A)).

Our laboratory had previously established the role of RhoC in inducing an IBC-like invasive phenotype in mammary epithelial cells [6]. We examined the new MCF10A-RhoC clones with a cell invasion assay, containing a Boyden chemotaxis chamber, with the bottom of the insert coated with reconstituted basement membrane matrix. We confirmed that MCF10A-RhoC WT exhibited two-fold increase of cell invasion, and 


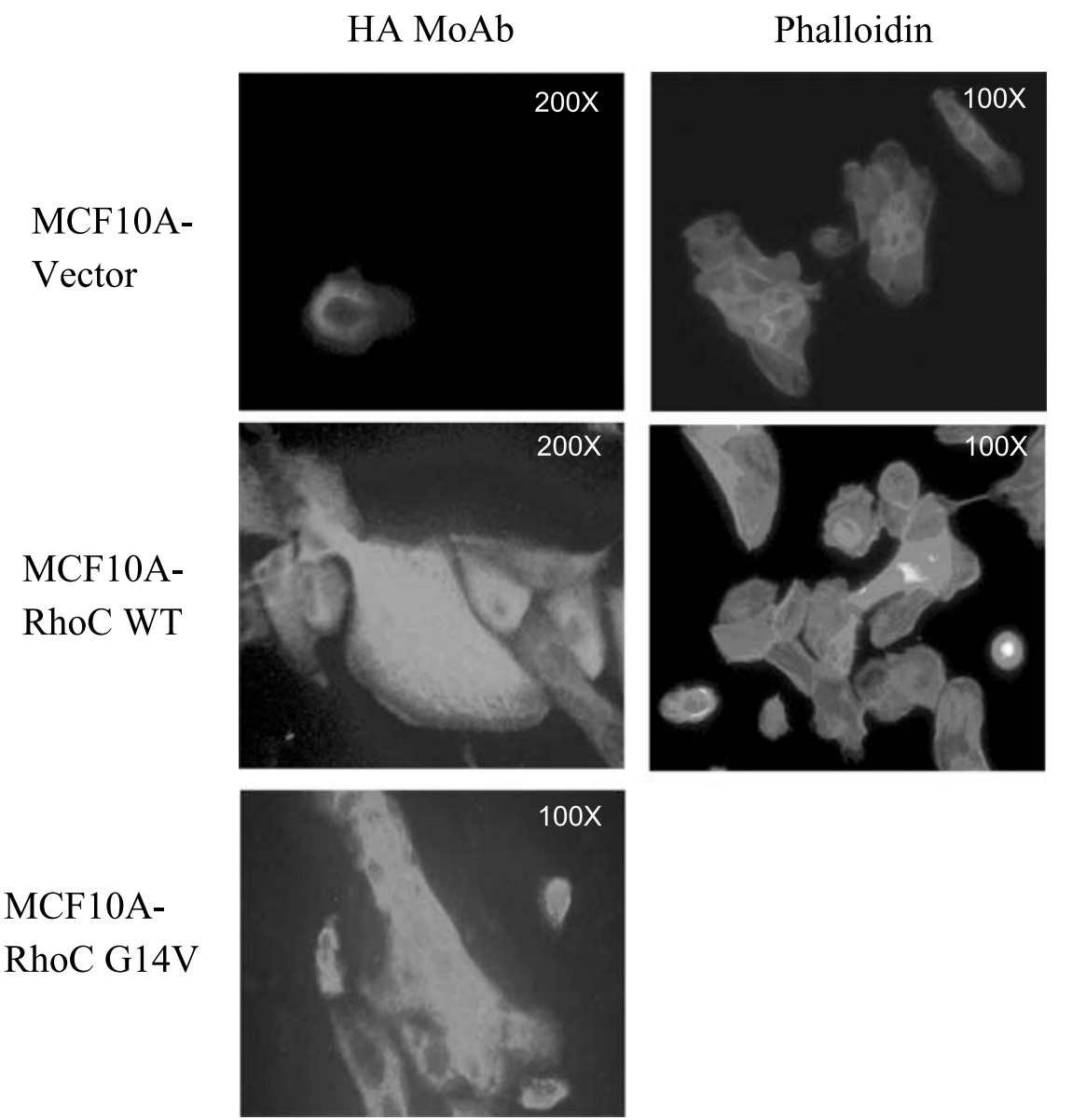

Figure 2. Fluorescence microscopy of the RhoC transfectants staining with the anti-HA monoclonal antibody (green) to visualize HA-RhoC, or rhodamine-phalloidin (red) to visualize stress fibers. Cells grown on chamber slides were fixed with 3\% paraformaldehyde, permeablized with $0.1 \%$ Triton X-100, and blocked with $1 \%$ BSA. The samples were incubated with either the primary anti-HA antibody and the Alexa Fluor 488-conjugated secondary antibody, or rhodamine-phalloidin.

the RhoC G14V cells had three-fold higher invasion than controls (Figure 3(B)).

\section{Microarray analysis of the MCF10A-RhoC WT/G14V transfectants}

Primary analysis of gene expression in MCF10ARhoC WT/G14V, along with MCF10A-vector as controls, was done using the Genepix software package (Axon Instruments, Union City, CA). Cy5 to Cy3 ratios were determined for the individual genes along with various other quality control parameters (e.g., intensity over local background). Bad spots and defective areas on the array were inspected and manually flagged. Spots with small diameters ( $<50$ microns) and spots with low signal strengths $<350$ fluorescence intensity units over local background in the more intense channel were also discarded. These data files were then imported into a Microsoft Access database and filtered for presence of data points across arrays and selected for change in expression levels by 2-fold or more.

SAM analysis [19] was performed by comparing gene expression profiles of four RhoC overexpressing clones (two MCF10A-RhoC WT and two MCF10AG14V clones) against two RhoC low-expressing clones. Data were normalized per array by multiplication by a factor to adjust the aggregate ratio of medians to one, then log base two transformed, and median centered. These normalized data were divided into two groups for comparison using a two-class, unpaired $t$-test. Critical values for the analysis include: Iterations $=200$, random number seed 80842225, a fold change cutoff of 1.5 and a delta cutoff of 0.598 , resulting in a final largest median false discovery 

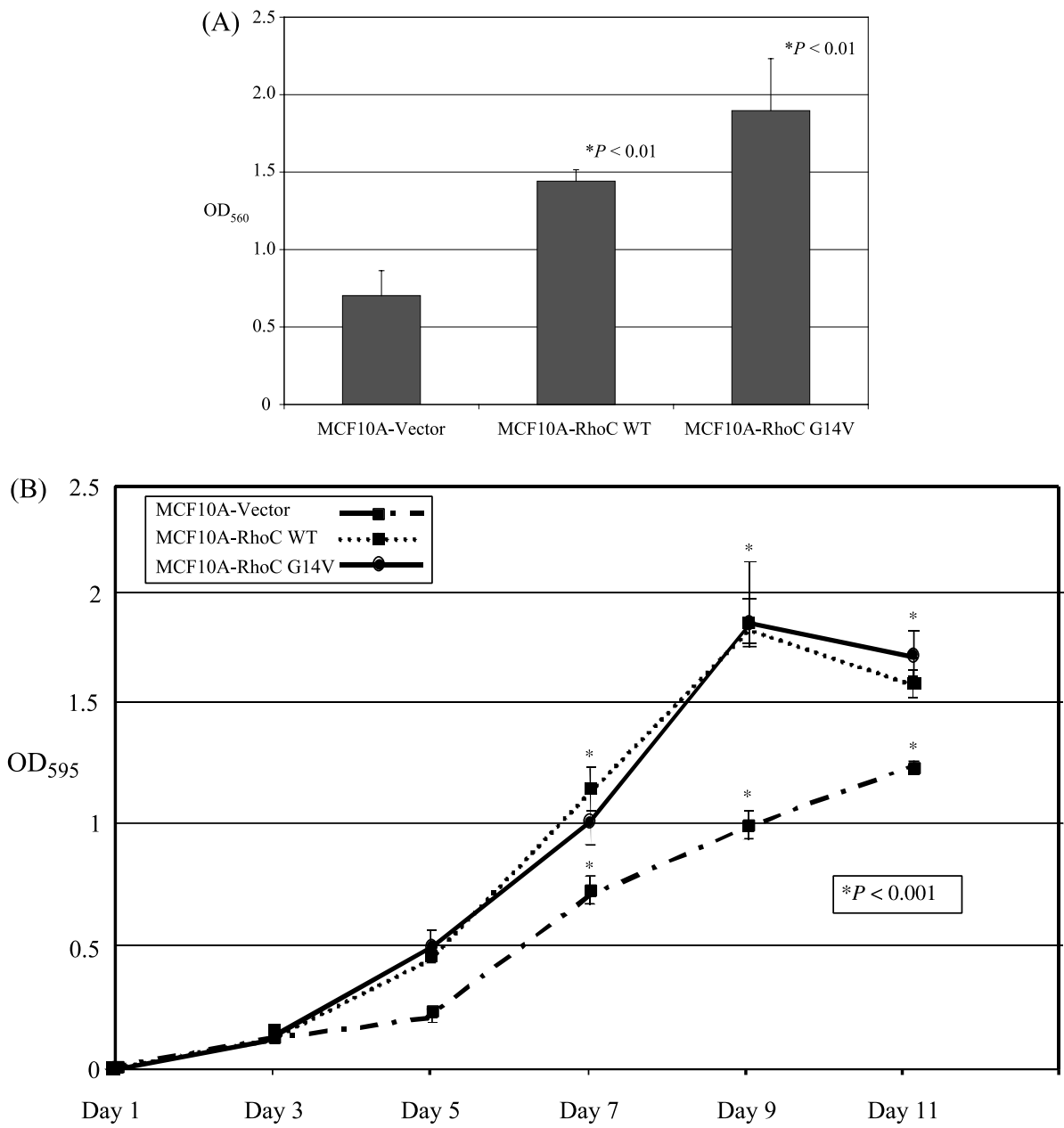

Figure 3. The RhoC overexpressing clones exhibit enhanced invasion and proliferation. (A) Quantitative invasion assay. The RhoC WT/G14V overexpressing cells were resuspended in serum-free medium and seeded in the chemotaxis chamber coated with basement membrane ECM. Cells penetrating through the artificial basement membranes were stained and resuspended in $10 \%$ acetic acid for $\mathrm{OD}_{560}$ readings. (B) Monolayer growth rate. RhoC transfectants (500 cells), taken at day 1,3,5, 7, 9, and 11, were incubated with MTT, solubilized in DMSO, and measured absorbance at $\mathrm{OD}_{595}$.

rate of $0.867 \%$ for the 115 genes selected as significant (108 relatively up- and seven relatively downregulated between RhoC overexpressing and RhoC low-expressing clones).

Overexpression of RhoC WT/G14V significantly altered message levels of genes involved in invasion and metastasis

In order to help elucidate the downstream signaling of RhoC GTPase, we have selected several significantly upregulated genes from the microarray results and examined their mRNA expression by quantitative RT-PCR. The housekeeping gene GAPDH served as a loading control (Figure 5(A and B)). We have found that the message of cyclin D1, a key regulator required for the G1/S transition in the cell cycle [20] was enhanced up to two-fold in the MCF10A-RhoC clones (Figure 5(A)). Overexpression of RhoC WT/G14V also increased the expression of two key angiogeneic factors, VEGF-C [21] and CXCL1 chemokine [22] (Figure 5(A)). Whereas we observed moderate stimulation of VEGF-C around two-fold, CXC chemokine ligand 1 (CXCL1) expression was highly enhanced to seven-fold in the MCF10A-RhoC WT clone compared to the vector-only controls (Figure 5(A)). The mRNA of Caveolin-2, a protein element of caveolar membrane [23], showed two-fold increase in the MCF10A-RhoC cells (Figure 5(A)). Notably as well, RhoC has a significant effect on the message level of 
fibronectin, a major component of connective-tissue type of ECM [24] (Figure 5(A)). Both alternatively spliced transcripts of fibronectin [25] were tested and they were equivalently upregulated. Fibronectin and CXCL1 are the two most upregulated genes identified in the array analysis.

On the other hand, we have found that RhoC overexpression decreased the message level of IGFBP-2, a high-affinity IGF binding protein. IGFBP2 is known to function as an inhibitor of IGF, a potent mitogen of breast cancer cells [26]. The mRNA of IGFBP-2 in the MCF10A-RhoC WT and MCF10A-G14V clones was lowered for 70 and 50\%, respectively (Figure 5(B)) which correlated with and confirmed the array data. This result further supports the role of RhoC in enhancing tumor aggressiveness and perhaps enabling enhanced IGF signaling.

\section{Discussion}

In this study, we summarize our initial work to explore the molecular basis for RhoC-induced invasive phenotype in spontaneous immortalized breast cells (MCF10A) using RNA microarray techniques. We have focused our validation experiments on five upregulated gene products involved in cell cycle progression (cyclin D1), angiogenesis (VEGF-C and CXCL1), and cell adhesion (fibronectin), and one down-regulated gene (IGFBP2).

Cyclin D1 is essential for G1 cell cycle progression and its expression is significantly higher in a variety of tumors, notably breast, parathyroid and sarcomas [27]. Inhibition of Rho, as well as Rac and Cdc42, can arrest the cell cycle at G1 [24]. Rac and Cdc42 signaling activate JNK and p38, which in turn stimulate cyclin D1 expression [28]. Conversely, activation of RhoA, a protein highly homologous to RhoC, stimulates transcription of AP-1, a major transcription factor controlling the cyclin D1 promoter [28]. Cyclin D1 transcription is also induced by the integrin-ECM signaling pathway [24]. Interactions between fibronectin and integrin activate focal adhesion kinase (FAK), which, in turn, transmits the signal to Rac and induces cyclin D1 transcription [24]. We have observed significant upregulation of fibronectin in our RhoC clones (Figure 5(A)). Therefore, increased cyclin D1 message in RhoC overexpressed breast cells may arise from the concerted effect of RhoC and Rac/Cdc42 signaling or possibly by the effects of fibronectin-dependent integrin activation.
VEGF-C, a member of the vascular endothelial growth factor family, is crucial for new capillary growth from existing vessels [21]. VEGF-C binds with high affinity to the VEGF receptor 3 (VEGFR3), but it also binds to VEGFR2 with low affinity [21]. VEGF/VEGFR signaling is essential for endothelial cell proliferation, chemotaxis, and vascular permeability. Mice carrying a VEGFR2 deletion did not have differentiated blood vessels or capillaries, whereas VEGFR3 knockout mice showed no defects in angiogenesis, but had abnormal vessel permeability causing fluid accumulation [21]. In previous work [29], we showed that RhoC activation induces angiogenesis, likely through p38 signaling. Now we show that the VEGF-C is specifically upregulated by RhoC activation, suggesting a possible role of RhoC also in promoting lymphagiogenesis.

CXCL1 is also known as melanoma growth regulated protein (GRO), as it was first identified from human melanoma conditioned medium [22]. CXC chemokines are heparin-binding proteins with two conserved cysteines flanking one other residue. The Nterminus of many family members (termed as ELR+) contains the ELR motif (Glu-Leu-Arg). The ELR+ chemokines, including CXCL1, are highly effective in promoting angiogenesis in physiological concentration of 1-100 Nm [30]. In melanoma, uncontrolled increases of CXCL1 or CXCL8 chemokines lead to angiogenesis and tumorigenesis, with concomitant constitutively activation of NF-kB. These CXCLoverexpressed melanoma cells also become greatly metastatic [22].

Fibronectin is a major component of stromal matrices, which are found at sites of injury and inflammation. Cell adhesion to this type of matrices often leads to migration and proliferation, instead of growth arrest and differentiation as occurs in contact with the basement membrane-type of ECM [31]. Transcription of fibronectin is stimulated by cAMP (forskolin) and TGF- $\beta$ [32]. TGF- $\beta$, a potent regulator for cell growth, activates RhoA-dependent signaling and mediates the epithelial-mesenchymal transition and stress fiber formation [33, 34]. In addition, expression of NET1, a Rho GEF, is induced by TGF- $\beta$ [35]. In contrast, cAMP activates cAMP-dependent kinase (PKA), which phosphorylates and inhibits RhoA [36]. Taken together based on these data, we propose that RhoC overexpression may help enhance TGF- $\beta$ signaling thereby enhancing fibronectin expression.

Our results also indicate that caveolin-2 is upregulated in the RhoC overexpressing breast cells 


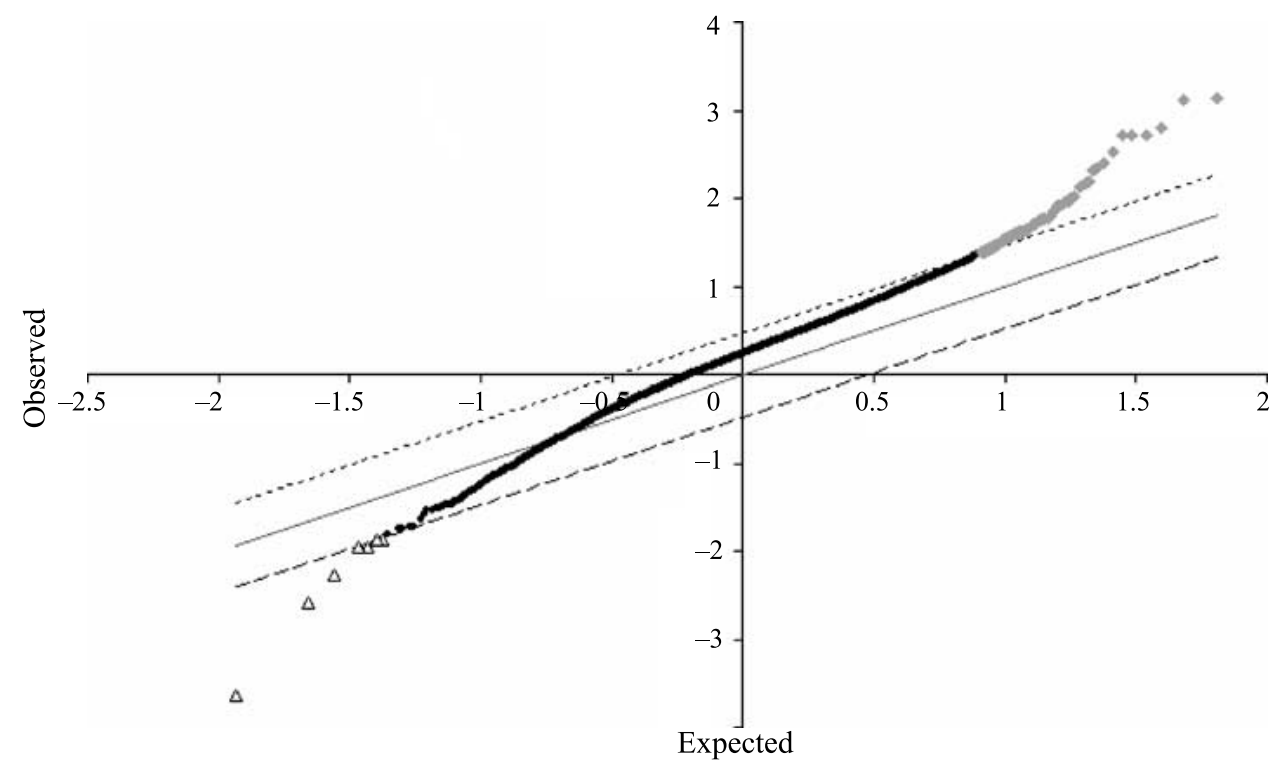

Figure 4. SAM analysis plot obtained by comparing gene expression profiles of two MCF10A-RhoC WT and two MCF10A-G14V clones against two RhoC low-expressing clones showing 108 genes as relatively up- and seven genes as relatively down-regulated at a false discovery rate of $0.867 \%$. Each spot on the plot represents one gene on the chip. The spots enclosed by the diagonal lines represent unchanged gene expression levels; the genes falling out of that range on the +ve plane are up-regulated (shown as red circles), and the genes falling out of that range on the - ve plane are down-regulated (shown as green triangles).

(Figure 5(A)). Caveolins are major structural proteins of caveolae, which are plasma membrane invaginations found in many cell types, including fibroblasts, epithelial cells and endothelial cells [37]. These membrane ruffles support and concentrate growth factor receptors and their immediate downstream signaling. The caveolin gene family consists of three members, caveolin-1, -2 , and -3 . Caveolin-1 is known to form homodimers or heterodimers with caveolin-2 in caveolae biosynthesis [37]. The function of caveolin-2 in human cancer is very poorly understood, compared to the family member caveolin-1. The caveolin1 gene is frequently deleted in human chromosome in a plethora of cancers, including squamous cell carcinomas, ovarian adenocarcinomas, prostate and breast cancers [37]. Down-regulation of caveolin-1 is also known as a feature of terminally differentiated cells [38]. Furthermore, the molecular mechanism of caveolin-1 as a tumor suppressor was to inhibit Neu tyrosine kinase pathway [39]. Overexpression of caveolin-1 inhibited the Neu pathway, and reduced tumor size in Neu-expressing transgenic mice. Interestingly, oncogenic activation of Neu down-regulated caveolin-1, but not caveolin-2 [39]. Since we have observed moderate increase of caveolin-2 in MCF10ARhoC, highly invasive breast cells (Figure 5(A)), it would be interesting to explore whether caveolin-
2 serves a specific role in tumorigenesis in this system.

In our array expression experiments, we have found 108 upregulated genes and only seven downregulated genes when we compared the MCF10ARhoC WT/G14V clones with MCF10A-Vector and RhoC low-expressing cells (Figure 4). We identified IGFBP-2 message levels to be lowered in both WT and G14V transfectants using quantitative RTPCR (Figure 5(B)). IGFBP2 is a negative regulator of the IGF system. IGF (insulin-like growth factors) are important mediators of cell growth and development. They are synthesized by nearly all tissues in the body and their functions are regulated by receptor tyrosine kinases in a complex circuitry of signaling. In breast cell carcinogenesis, IGF-1 increases cancer cell proliferation through two mechanisms. IGF1 enhances cancer cell growth and also promotes stromal cell outgrowth in a paracrine or autocrine manner [26]. IGFBP3, also a high-affinity inhibitor to IGFs, repressed IGF signaling in breast cancer cells even without physical interactions with IGF [40]. Furthermore, WISP3/LIBC, a tumor suppressor first identified in our laboratory, was predicted as a member of IGFBP-rPs by its protein sequence [5]. IGFBP-rP proteins show relatively low affinity for IGF binding so they may regulate cell growth by mechanisms other 


\section{Cyclin D1}

Vector WT CA

Caveolin-2

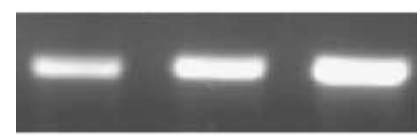

VEGF-C

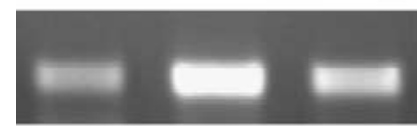

GAPDH

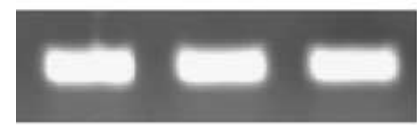

Fibronectin

GAPDH

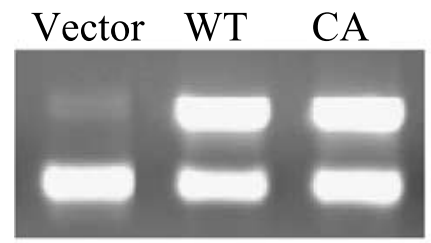

CXCL1

GAPDH

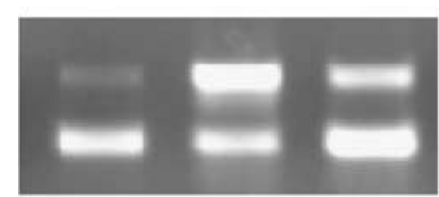

(B)

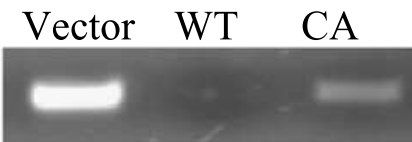

\section{GAPDH}

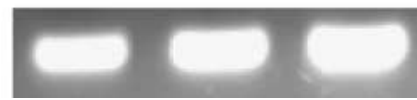

Figure 5. Quantitative RT-PCR analysis of the RhoC clones. Sequence-specific primer pairs were designed to amplify cyclin D1, caveolin-2, VEGF-C, fibronectin, CXCL1, and IGFBP2 (the mRNA sequences of the above genes were downloaded from Genbank, NCBI, NIH). In the RT-PCR reaction, $100 \mathrm{mg}$ of cDNA was amplified with $25 \mathrm{ng}$ each of the primers. The products were separated on a $1.2 \%$ agarose gel. GAPDH was served as a loading control.

than direct binding to IGF. IGFBP-rP1 was also proposed to be a potential tumor suppressor protein for prostate cancer [26]. In conclusion, down-regulation of IGFBP2 by RhoC supports that RhoC overexpression may enhance an IBC-like, invasive phenotype in normal breast cells by modulation of IGF signaling. Taken together, our data present a number of key molecules that are likely to be involved in the phenotype changes induced by RhoC, suggesting future investigations in this area.

\section{Acknowledgements}

Work supported by grants from Department of Defense Breast Cancer Res Program (DAMD 17-00-1-0345), National Institute of Health (R01 CA77612), NIH Head and Neck SPORE grant (P50 CA097248/SPORE), and Susan G. Komen Breast Cancer Res Foundation to S.D.M.

\section{References}

1. Jaiyesimi IA, Buzdar AU, Hortobagyi G: Inflammatory breast cancer: a review. J Clin Oncol 10: 1014-1024, 1992

2. Lee BJ, Tannenbaum NE: Inflammatory carcinoma of the breast: a report of twenty-eight cases from the breast clinic of Memorial Hospital. Surg Gynecol Obstet 39: 580-595, 1924

3. Merajver SD, Weber BL, Cody R, Zhang D, Strawderman M, Calzone KA, LeClaire V, Levin A, Irani J, Helvie M, August D, Wicha MS, Lichter A, Pierce LJ: Breast conservation and prolonged chemotherapy for locally advanced breast cancer: the University of Michigan experience. J Clin Oncol 15: 2873-2881, 1997

4. Swain SM, Sorace RA, Bagley CS, Danforth DN, Bader J, Wesley MN, Steinberg SM, Lippman ME: Neoadjuvant chemotherapy in the combined modality approach of locally advanced nonmetastatic breast cancer. Cancer Res 47: 3889-3894, 1987

5. van Golen KL, Davies S, Wu ZF, Wang Y, Bucana CD, Root H, Chandrasekharappa S, Strawderman M, Ethier SP, Merajver SD: A novel putative low-affinity insulin-like growth factorbinding protein, LIBC (lost in inflammatory breast cancer), and RhoC GTPase correlate with the inflammatory breast cancer phenotype. Clin Cancer Res 5: 2511-2519, 1999

6. van Golen KL, Wu ZF, Qiao XT, Bao LW, Merajver SD: RhoC GTPase, a novel transforming oncogene for human mammary epithelial cells that partially recapitulates the inflammatory breast cancer phenotype. Cancer Res 60: 5832-5838, 2000

7. Kleer CG, Zhang Y, Pan Q, van Golen KL, Wu ZF, Livant D, Merajver SD: WISP3 is a novel tumor suppressor gene of inflammatory breast cancer. Oncogene 21: 3172-3180, 2002

8. Ethier SP, Mahacek ML, Gullick WJ, Frank TS, Weber BL: Differential isolation of normal luminal mammary epithelial cells and breast cancer cells from primary and metastatic sites using selective media. Cancer Res 53: 627-635, 1993

9. Etienne-Manneville S, Hall A: Integrin-mediated activation of Cdc42 controls cell polarity in migrating astrocytes through PKCzeta. Cell 106: 489-498, 2001

10. Kamai T, Tsujii T, Arai K, Takagi K, Asami H, Ito Y, Oshima $\mathrm{H}$ : Significant association of Rho/ROCK pathway with invasion and metastasis of bladder cancer. Clin Cancer Res 9: 2632-2641, 2003

11. Horiuchi A, Imai T, Wang C, Ohira S, Feng Y, Nikaido T, Konishi I: Up-regulation of small GTPases, RhoA and RhoC, is associated with tumor progression in ovarian carcinoma. Lab Invest 83: 861-870, 2003 
12. Suwa H, Ohshio G, Imamura T, Watanabe G, Arii S, Imamura M, Narumiya S, Hiai H, Fukumoto M: Overexpression of the $r h o C$ gene correlates with progression of ductal adenocarcinoma of the pancreas. Br J Cancer 77: 147-152, 1998

13. Clark EA, Golub TR, Lander ES, Hynes RO: Genomic analysis of metastasis reveals an essential role for RhoC. Nature 406: 532-535, 2000

14. Soule HD, Maloney TM, Wolman SR, Peterson Jr WD, Brenz R, McGrath CM, Russo J, Pauley RJ, Jones RF, Brooks SC: Isolation and characterization of a spontaneously immortalized human breast epithelial cell line, MCF-10. Cancer Res 50: 6075-6086, 1990

15. Soule H, McGrath CM: Immortal human mammary epithelial cell lines. Primary Examiner: Kepplinger, Esther L. Assistant Examiner: Scheiner, Toni R. Attorney AoFK, Robert L. G, Dykema (eds). 317610[5,026,637]. 6-25-1991. Michigan/United States, Jones et al., Breast Cancer Res Group and Pathology Dept., Michigan Cancer Foundation, Detroit, MI, Proceedings of AACR, Vol. 29, March 1988

16. Kleer CG, van Golen KL, Zhang Y, Wu ZF, Rubin MA, Merajver SD: Characterization of RhoC expression in benign and malignant breast disease: a potential new marker for small breast carcinomas with metastatic ability. Am J Pathol 160: 579-584, 2002

17. Dhanasekaran SM, Barrette TR, Ghosh D, Shah R, Varambally S, Kurachi K, Pienta KJ, Rubin MA, Chinnaiyan AM: Delineation of prognostic biomarkers in prostate cancer. Nature 412: 822-826, 2001

18. Microarrays.org is a public source for microarray protocols and software, 2003. www.microarrays.org

19. Tusher VG, Tibshirani R, Chu G: Significance analysis of microarrays applied to the ionizing radiation response. Proc Natl Acad Sci USA 98: 5116-5121, 2001

20. Xiong Y, Connolly T, Futcher B, Beach D: Human D-type cyclin. Cell 65: 691-699, 1991

21. Zachary I: Signaling mechanisms mediating vascular protective actions of vascular endothelial growth factor. Am J Physiol Cell Physiol 280: C1375-C1386, 2001

22. Dhawan P, Richmond A: Role of CXCL1 in tumorigenesis of melanoma. J Leukoc Biol 72: 9-18, 2002

23. Couet J, Belanger MM, Roussel E, Drolet MC: Cell biology of caveolae and caveolin. Adv Drug Deliv Rev 49: 223-235, 2001

24. Danen EH, Yamada KM: Fibronectin, integrins, and growth control. J Cell Physiol 189: 1-13, 2001

25. Norton PA, Hynes RO: In vitro splicing of fibronectin premRNAs. Nucl Acids Res 18: 4089-4097, 1990

26. Moschos SJ, Mantzoros CS: The role of the IGF system in cancer: from basic to clinical studies and clinical applications. Oncology 63: 317-332, 2002

27. Polsky D, Cordon-Cardo C: Oncogenes in melanoma. Oncogene 22: 3087-3091, 2003

28. Sahai E, Marshall C: RHO-GTPases and Cancer. Nature Rev - Cancer 2: 133, 2002
29. van Golen KL, Bao L, Pan Q, Miller FR, Wu ZF, Merajver $\mathrm{S}$ : Mitogen activated protein kinase pathway is involved in RhoC GTPase induced motility, invasion and angiogenesis in inflammatory breast cancer. Clin Exp Metast 19: 301-311, 2002

30. Strieter RM, Belperio JA, Keane MP: CXC chemokines in angiogenesis related to pulmonary fibrosis. Chest 122: 298S-301S, 2002

31. Boudreau N, Bissell MJ: Extracellular matrix: the networking solution. In: Alison MA (ed) The Molecular Basis of Cell and Tissue Organisation. Nature Publishing Group, London, New York and Tokyo, 2002, pp 209-224

32. Dean DC, Newby RF, Bourgeois S: Regulation of fibronectin biosynthesis by dexamethasone, transforming growth factor beta, and cAMP in human cell lines. J Cell Biol 106: 2159-2170, 1988

33. Bhowmick NA, Ghiassi M, Bakin A, Aakre M, Lundquist CA, Engel ME, Arteaga CL, Moses HL: Transforming growth factor-beta1 mediates epithelial to mesenchymal transdifferentiation through a RhoA-dependent mechanism. Mol Biol Cell 12: 27-36, 2001

34. Edlund S, Landstrom M, Heldin CH, Aspenstrom P: Transforming growth factor-beta-induced mobilization of actin cytoskeleton requires signaling by small GTPases Cdc42 and RhoA. Mol Biol Cell 13: 902-914, 2002

35. Shen X, Li J, Hu PP, Waddell D, Zhang J, Wang XF: The activity of guanine exchange factor NET1 is essential for transforming growth factor-beta-mediated stress fiber formation. J Biol Chem 276: 15362-15368, 2001

36. Ellerbroek SM, Wennerberg K, Burridge K: Serine phosphorylation negatively regulates RhoA in vivo. $\mathrm{J}$ Biol Chem 278: 19023-19031, 2003

37. Razani B, Lisanti MP: Caveolin-deficient mice: insights into caveolar function human disease. J Clin Invest 108: 1553-1561, 2001

38. Campbell L, Gumbleton M, Ritchie K: Caveolae and the caveolins in human disease. Adv Drug Deliv Rev 49: 325-335, 2001

39. Engelman JA, Lee RJ, Karnezis A, Bearss DJ, Webster M, Siegel P, Muller WJ, Windle JJ, Pestell RG, Lisanti MP: Reciprocal regulation of neu tyrosine kinase activity and caveolin-1 protein expression in vitro and in vivo. Implications for human breast cancer. J Biol Chem 273: 20448-20455, 1998

40. Oh Y, Muller HL, Lamson G, Rosenfeld RG: Insulinlike growth factor (IGF)-independent action of IGF-binding protein-3 in Hs578T human breast cancer cells. Cell surface binding and growth inhibition. J Biol Chem 268: 14964-14971, 1993

Address for offprints and correspondence: Sofia D. Merajver, MD, $\mathrm{PhD}$, Department of Internal Medicine, Division of Hematology and Oncology, University of Michigan Medical School, $1500 \mathrm{E}$. Medical Center Drive, Ann Arbor, MI 48109-0948, USA; E-mail: smerajve@umich.edu 